\title{
Les agglomérations transfrontalières: des systèmes urbains en voie d'intégration? Les espaces urbains de la «frontière» du territoire français
}

\section{Bernard Reitel, Mulhouse}

\section{Introduction}

En tant que «limite politique signifiante» (Piermay 2005), la frontière a joué un rôle majeur dans la construction du territoire national de la France (NoRdman 1998). Elle apparaît comme une discontinuité politique majeure, mais en même temps présente une grande porosité. Au niveau local, le jeu des acteurs économiques et sociaux est souvent dynamique: ainsi sur la frontière du territoire français, des espaces urbains transfrontaliers se sont constitués. Traversées par une ou plusieurs frontières d'Etat, les agglomérations transfrontalières forment des objets géographiques singuliers. Une première interrogation porte sur la porosité de la frontière et le degré d'intégration de ces espaces urbains.

Dans le cadre de la construction européenne, la dévaluation des frontières est devenue une réalité (FoucHER 2000). Encouragées par l'Union Européenne (UE), de nombreuses collectivités urbaines frontalières ont élaboré des projets portant sur des périmètres transfrontaliers et impliquant des coopérations. Une deuxième interrogation porte sur les logiques de projets: aident-elles à construire une dimension territoriale transcendant la frontière?

L'objectif de cet article consiste à évaluer le degré d'intégration de quinze agglomérations situées sur les frontières de France en tenant compte à la fois d'une dimension spatiale (organisation, pratiques) et d'une dimension territoriale (politiques de coopération, projets). «L'intégration peut être définie comme la mise en place d'une interdépendance étroite entre les parties d'un tout» (Françols et al. 2002: 355). L'hypothèse est que l'apparition de la dimension supranationale conduit à une recomposition de la frontière, c'est-à-dire à une mutation de ses propriétés. L'objectif est à la fois méthodologique (élaborer une grille d'analyse qui permette de mesurer les formes d'intégration) et théorique (apporter une meilleure connaissance de la frontière à travers la mesure d'intégration).

\section{La frontière et les espaces urbains aux frontières de France}

\subsection{La frontière d'Etat, un objet ambivalent}

En dehors de quelques exceptions (GUICHONNET \& RAFFESTIN 1974; PrescotT 1978), la frontière a été longtemps peu étudiée. A partir des années 1980, la signature de l'Acte Unique suscite un regain d'intérêt et porte sur plusieurs aspects. En tant que limite de souveraineté, la frontière renvoie à l'exclusivité territoriale de l'Etat-nation (ANDERSON 1996): elle est un objet d'affirmation de la puissance de l'Etat (FouCHER 1986) et est liée à l'émergence d'une conscience nationale et à l'édification du territoire national (PAASI 1996). La frontière est un «objet qui met de la distance dans la proximité géographique» (ARBARET-Schulz et al. 2004: 8).

L'ambivalence de la frontière est largement soulignée, notamment dans d'autres disciplines qui utilisent ce concept (Newman 2003; Pollmann 1999). En géographie aussi, elle est à la fois coupure et couture, ligne qui relie deux territoires nationaux (COURLET 1988; LeIMGRUBER 1989). Autour de la frontière se construit un espace spécifique qui structure autant les populations que les relations (DonNan \& WiLson 1999).

D'après RAFFESTIN (1986: 4), la frontière est un objet complexe qui possède quatre propriétés: l'affirmation, la régulation, le différentiel et la relation. Elle est à la fois «l'expression d'un pouvoir en acte», un instrument de régulation (qui opère un filtrage), un objet de différenciation (p.ex. entre deux systèmes politiques, juridiques, culturels) et un élément de relation (la contiguïté de deux territoires produit des relations licites et illicites). En France, quels que soient les régimes politiques, la frontière a été instrumentalisée par l'Etat (Nordman 1998). Des effets-frontière sont cependant observés en fonction du degré d'ouverture (RENARD \& Picouet 1993). Ils traduisent l'apparition de «milieux frontaliers» (Martinez 1994: 8) qui présentent de sensibles différences avec le reste du territoire national en raison de leur confrontation à l'international de proximité.

Des tentatives de modélisation des interactions spatiales transfrontalières ont été entreprises qui portent soit sur des pratiques sociales (MARTINEZ 1994), soit sur des flux économiques (RatTI \& Reichman 1993). Dans le premier cas, quatre types ont été décrits: l'aliénation (relations réduites), la coexistence (relations au niveau transnational principalement), l'interdépendance (le système est ouvert et permet de nombreux flux qui débouchent sur des complémentarités), l'intégration (la frontière s'estompe comme barrière et comme élément de différenciation). Ces deux tentatives sont envisagées comme des modèles temporels en partant d'un état où la frontière est relativement fermée, à un 
autre, où elle est complètement ouverte. C'est de façon schématique l'évolution qu'ont connues les frontières des Etats de l'Union Européenne depuis la Seconde Guerre mondiale. En tant que membre fondateur de la Communauté économique européenne (CEE), la France est largement associée à ce processus d'intégration qui a connu plusieurs étapes (WASTL-WALTER \& KOFLER 2000). Des études empiriques ont montré les effets de ce processus sur les dynamiques transfrontalières (ANDERSON 1983; SCHNEIDER-SLIWA 2002).

\subsection{Des espaces urbains transfrontaliers aux frontières de France}

Les nombreuses analyses entreprises sur les espaces urbains localisés sur des frontières politiques (Ehlers 2002; Ginet 1994; Jouve 1994; WaACK 2000) insistent sur le caractère prégnant de la frontière. Certains auteurs ont proposé une perspective historique (Mensot 1996), ou géographique (Gradus \& Lithwick 2001; Reitel et al. 2002), mais aussi géopolitique (Kotek 1996). L'affirmation du pouvoir politique est particulièrement visible sur la frontière du territoire français: elle s'inscrit dans le dispositif de défense complexe et hiérarchisé construit à la fin du $\mathrm{XVII}{ }^{\text {ème }}$ siècle, dans les fonctions et dans les paysages urbains (MEYER 1984). Mais la ville fortifiée est aussi instrumentalisée: elle est un lieu de représentation du pouvoir, une vitrine à l'usage des territoires voisins (DENIS 2002).

Le déplacement de dyades (implantation d'une limite internationale dans un tissu urbain existant) correspond à des cas exceptionnels tels que Sarrebruck ou Strasbourg. En général, le processus d'urbanisation résulte de l'activation de propriétés de la frontière parmi lesquelles l'exploitation des différentiels par les acteurs économiques semble déterminante. Ceux-ci tirent profit de la proximité d'un territoire disposant d'un système de normes différent (RAFFESTIN 1980) et y implantent un établissement. Un processus d'essaimage est notamment observable autour de Bâle et de Genève (Bergier 1983; Staehlin 1979). Les différences entre les normes techniques provoquent également des ruptures de charge qui sont créatrices d'activités économiques (MARCOU \& JACQUOT 1997). Dans le cas de Lille, dès la fin du XIX ${ }^{\text {ème }}$ siècle, la ville attire une main d'œuvre en provenance de la Belgique (LENTACKER 1974). Tout en assurant sa fonction de filtrage, l'Etat mène une politique d'ouverture qui se traduit par l'émergence de relations durables entre les acteurs économiques. L'Etat exerce avant tout son contrôle sur les collectivités qui ont peu de relations avec les pouvoirs locaux étrangers contigus.

Cependant, ces espaces urbains, bien qu'animés par de nombreux flux, ne forment pas des systèmes urbains. Les flux (pratiques spatiales) s'organisent en fonction de deux paramètres: d'une part des polarités selon une logique de centre-périphérie à partir du pôle principal, d'autre part des différentiels qui induisent des spécialisations et des complémentarités (SANDTNER \& EDER 2000). Le caractère polycentrique est affirmé: les centralités s'expriment par des fonctions urbaines (commerces, services, équipements culturels) et par la présence de pouvoirs publics qui ont à cœur de se singulariser par rapport aux collectivités du pays voisins (REITEL 2006). La frontière souligne les identités: la traversée continue d'être associée au passage à l'altérité, malgré la continuité du tissu urbain. L'agglomération transfrontalière est composée de territoires urbains juxtaposés entre lesquelles existent des interactions, mais qui sont soumis à des temporalités différentes (SAEZ et al. 1997). Même si la frontière constitue un objet partagé, l'agglomération n'est pas une entité politique, ni une entité aisément identifiable. Contrairement aux agglomérations situées dans un cadre national unique, aucune autorité n'est habilitée à réguler cette différenciation, alors que dans un contexte national, l'Etat incite à penser la totalité de l'espace urbain en vertu de la continuité territoriale. Dans le contexte français, l'affirmation et la régulation semblent être les principales propriétés. Mais dans le même temps, les différences exploités par les acteurs économiques sont des facteurs d'urbanisation et par conséquent d'intégration. Les relations restent l'apanage de l'Etat central ou des acteurs économiques et sont transnationales bien plus que transfrontalières. Les collectivités françaises sont longtemps restées dans une logique de coexistence par rapport aux collectivités étrangères voisines.

\section{Méthodologie: mise en évidence des critères d'intégration}

L'agglomération reconnue par les autorités est frontalière, non transfrontalière: les espaces urbains sont définis par des critères nationaux. La base de données Geopolis présente l'avantage de pouvoir identifier toutes les agglomérations de plus de 10000 habitants en Europe (Moriconi-Ebrard 2002). En France, treize agglomérations sont localisées sur les bordures du territoire métropolitain, mais quinze ont été retenues dans l'analyse. En effet, sur la Côte Basque et la Riviera, deux niveaux ont été pris en compte:l'agglomération au sens restreint (Irun-Fuentarrabia-Hendaye, MonacoMenton-Ventimiglia) et l'ensemble linéaire qui s'étend sur plusieurs dizaines de kilomètres le long du littoral afin de tenir compte des dynamiques d'urbanisation des deux dernières décennies. Chaque espace urbain correspond à une construction spatiale et temporelle spécifique. Les quinze unités spatiales présentent une diversité de taille et de forme ainsi que dans la répartition des populations selon leur composante nationale respective (Figure 1). Ainsi, l'agglomération «polycen- 


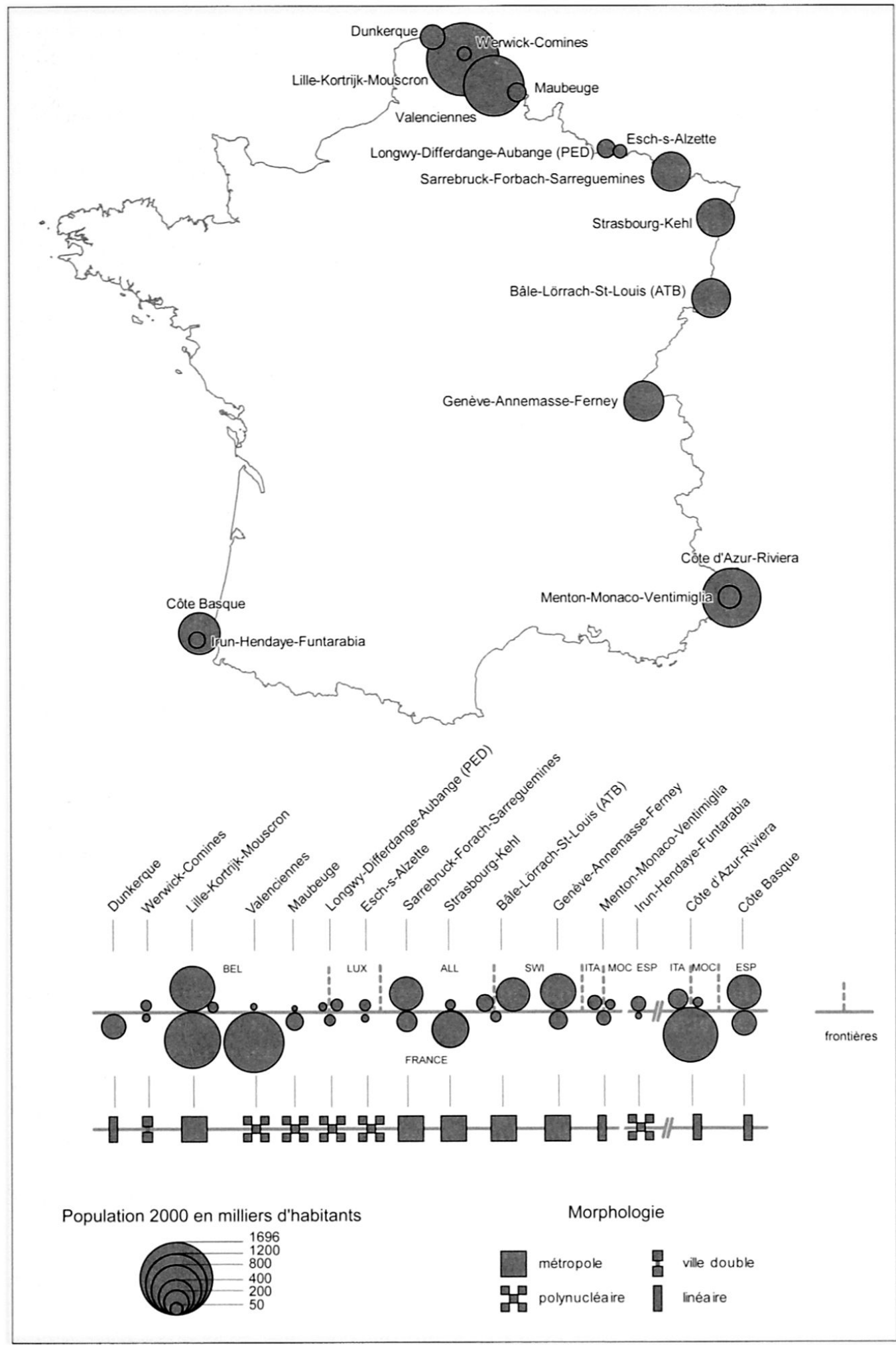

Fig. 1: Agglomérations transfrontalières «françaises» en 2000: population totale, population des composantes nationales et morphologie

"Französische» grenzüberschreitende Stadträume in 2000: Gesamtbevölkerung, Bevölkerung nach Herkunftsland und Stadtraumtypen

"French" cross-border agglomerations in 2000: total poupulation, population according to land of origin and agglomeration types

Sources: D'après Base Geopolis 2000, Mission Opérationnelle Transfrontalière (MOT); concept: B. Reitel; graphique: Centre de Recherches sur les Economies, les Sociétés, les ArTS et les Techniques (CRESAT), J.-P. Droux 


\begin{tabular}{|c|c|c|c|c|c|}
\hline & Variables & 0 & 1 & 2 & 3 \\
\hline \multirow[t]{6}{*}{$\begin{array}{l}\text { Intégration } \\
\text { spatiale }\end{array}$} & $\begin{array}{l}\text { Organisation } \\
\text { spatiale (OS) }\end{array}$ & $\begin{array}{l}\text { Absence de } \\
\text { relations }\end{array}$ & $\begin{array}{l}\text { Asymétrique } \\
\text { dominant }\end{array}$ & Polarisé & Polycentrique \\
\hline & Navetteurs (NV) & Néant & Faible & Moyen & Fort \\
\hline & Pratiques (PR) & Néant & Faible & Complémentaire & Interdépendance \\
\hline & \begin{tabular}{|l} 
Lieux \\
transfrontaliers \\
(LE)
\end{tabular} & Néant & 1 ou 2 & Plusieurs & Entité globale \\
\hline & Identité (ID) & Ignorance & Conflictuelle & Interdépendance & Partagée \\
\hline & $\begin{array}{l}\text { Réseaux collectifs } \\
\text { de transport (RE) }\end{array}$ & Néant & 1 ligne & Interconnection & Intégration \\
\hline \multirow[t]{6}{*}{$\begin{array}{l}\text { Intégration } \\
\text { territoriale }\end{array}$} & $\begin{array}{l}\text { Ancienneté } \\
\text { coopération (AN) }\end{array}$ & En cours & Moins de 15 ans & $15-25$ ans & Plus de 25 ans \\
\hline & Nom (NO) & Non & Oui & & \\
\hline & Vision (VI) & Non & \begin{tabular}{|l|} 
En cours \\
\end{tabular} & Partiel & Global \\
\hline & \begin{tabular}{|l|} 
Structure \\
d'encadrement \\
(ST)
\end{tabular} & Néant & Plate-forme & Association & $\begin{array}{l}\text { Structure } \\
\text { politique }\end{array}$ \\
\hline & Ressources (RE) & Néant & \begin{tabular}{|l|} 
Coopération \\
entre entités \\
existantes
\end{tabular} & $\begin{array}{l}\text { Structure peu } \\
\text { autonome }\end{array}$ & $\begin{array}{l}\text { Structure } \\
\text { autonome }\end{array}$ \\
\hline & $\begin{array}{l}\text { Projets } \\
\text { opérationnels (OP) }\end{array}$ & Néant & Technique & $\begin{array}{l}\text { Variété, phase de } \\
\text { faisabilité }\end{array}$ & $\begin{array}{l}\text { Variété, } \\
\text { réalisations }\end{array}$ \\
\hline
\end{tabular}

Tab. 1: Typologie de l'intensité de la coopération dans les agglomérations transfrontalières Typologie der Intensität der grenzüberschreitenden Kooperationen in Grenzstädten Typology of the intensity of cross-border co-operation in border cities Sources: Mission Opérationnelle Transfrontalière (MOT), sites internet des collectivités

trique» est caractérisée par une urbanisation à partir de plusieurs noyaux. Le type métropolitain correspond à une agglomération où un pôle exerce des fonctions de métropole régionale ou internationale.

L'absence de base homogène de données incite à créer sa propre base en sélectionnant plusieurs variables. La Mission Opérationnelle Transfrontalière (MOT) constitue la principale source d'information: créée par l'Etat français en 1998, elle a pour mission d'assurer des conseils auprès des collectivités territoriales pour leurs projets transfrontaliers et recueille de nombreux documents. La base de données est construite à partir de l'exploitation des documents consultés à la MOT. Les informations qualitatives sont transformées en informations quantitatives.

L'objectif est d'évaluer le degré d'intégration de ces espaces urbains, c'est-à-dire de voir si au niveau local, la frontière apparaît plus comme un objet de relation qu'un objet d'affirmation et de régulation.

"Intégrer est donc mettre en connexion, assurer des interrelations, effacer les ruptures et les distances entre des éléments qui conservent néanmoins leur être» (BRUNET 1997: 11).

Deux types de critères ont été identifiés: ils s'appuient sur des indicateurs utilisés par la Mission OpérATION-
Nelle Transfrontalière (MOT, 2003) pour effectuer une typologie portant sur l'intensité de la coopération dans les agglomérations. Les premiers cherchent à traduire l'intégration spatiale de l'agglomération (p. ex. organisation spatiale, pratiques, navetteurs), l'existence d'une réalité urbaine transfrontalière. Les seconds prennent en compte une dimension territoriale au sens d'un cadre d'actions politiques (p. ex. projets Interreg, types d'actions). Dans chaque agglomération, une valeur comprise entre 0 et 3 est attribuée à chaque critère (sauf lorsque le choix réside entre absence et présence). Les valeurs sont attribuées en fonction de l'ensemble des agglomérations et non dans l'absolu. Des informations complémentaires ont été cherchées sur les sites internet des collectivités, sur celui présentant les projets Interreg ou dans des organismes officiels (Institut National de la Statistique et des Etudes Economiques (INSEE) pour les navetteurs). Les valeurs reposent sur la typologie de Martinez (1994): le point le plus élevé est attribué lorsque existe une dimension supranationale, puis viennent dans l'ordre l'interdépendance, la coexistence et l'absence. Plus la frontière exerce un caractère de frein, plus le nombre de point est faible. Dans certains cas, les points sont donnés en fonction du degré de complexité: la valeur de l'organisation polycentrique est supérieur à celle de l'organisation polarisée (Tableau 1). 
Une analyse multivariée est réalisée sur ces informations qualitatives et hiérarchisées. L'analyse des correspondances multiples a pour objectif d'établir les relations non linéaires entre les modalités (variables valuées) et les unités spatiales et de présenter les proximités entre ces dernières. L'analyse présente des facteurs qui correspondent à une synthèse des informations essentielles. L'intensité des relations est révélée par la proximité entre les modalités et les unités spatiales sur des graphiques qui représentent les axes deux à deux (Escoffier \& PAGEs 1988).

\section{Des situations d'intégration entre coexistence et interdépendance}

Les deux premiers axes apportent $59,9 \%$ d'explication (Figure 2). Le premier axe oppose deux types de modalités: celles qui correspondent à une faible intégration spatiale (coexistence liée à la fonction de régulation de la frontière) à celles où existe une intégration de type territoriale (relations de coopération à un niveau local). Le deuxième axe oppose des interactions basées sur des pratiques transfrontalières et l'émergence d'une identité à des interactions reposant sur des différentiels (importance des navetteurs). Le troisième axe apporte peu d'explications et les oppositions sont moins marquées.

L'analyse fait apparaître trois informations principales. D'une part, elle montre que la dimension territoriale est d'autant plus forte que l'intégration spatiale est importante. D'autre part, des différences notables existent entre les agglomérations. Enfin, certaines variables d'intégration territoriale se révèlent particulièrement discriminantes (ancienneté de la coopération. vision,structure d'encadrement). Une classification ascendante hiérarchique est réalisée afin de répartir les 15 agglomérations dans un certain nombre de groupes les plus homogènes possibles. Un indice de dissimilarité est calculé entre les valeurs de chaque variable pour chaque unité spatiale (CARPENTIER 2005). Les valeurs des deux variables les plus proches sont regroupées. Cette opération est réitérée sur toutes les variables. Un dendrogramme (arbre hiérarchique) montre le degré de proximité existant entre chaque variable. L'indice de dissimilarité choisi est la distance euclidienne. Le dendrogramme établit clairement la différence entre deux types d'agglomérations: celles où la dimension territoriale est faible (coexistence des territoires), voire absente et celle où elle existe (interdépendance). Cependant, ce premier découpage est affiné. Cinq types sont décelés (Figure 3) et sont analysés en fonction des propriétés décrites par Martinez (1994) et par RafFestin (1986).

Un premier type décrit des espaces urbains caractérisés à la fois par une faible intégration spatiale et l'absence d'intégration territoriale. Dans le deuxième type, la dimension territoriale est plus forte ce qui montre qu'une coopération est engagée, mais elle reste réduite: p. ex. projet en phase initiale, absence de cadrage juridique, pas ou peu de projets opérationnels. Les territoires sont en phase de coexistence plus que d'interdépendance. Il s'agit pour l'essentiel d'agglomérations présentant une large extension spatiale (agrégats plus ou moins linéaires étendus sur plusieurs dizaines de kilomètres et dont la composante transfrontalière est très faible). Les pôles urbains principaux sont ainsi éloignés de la frontière (Valenciennes se trouve à plus de 10 kilomètres de la frontière par exemple). Dans les autres cas, les agglomérations sont plus petites et centrées sur la frontière, mais la dimension politique a été peu développée jusqu'à présent soit parce que l'entité fait partie d'une composante plus importante (Werwick-Comines au projet piloté par Lille), soit parce que la volonté politique locale est faible (frontière franco-italienne).

Dans les trois types suivants, la dimension territoriale est toujours présente, mais elle présente des aspects différenciés:

- Dans le premier cas, la démarche de coopération a été engagée dans les années 1990 et s'avère particulièrement intense: c'est au Pays Basque, que les structures d'encadrement politiques les plus intégrées ont été créées (Consorcio Bidassoa en 1998, Eurocité Basque en 2000). Une forte volonté politique est à l'origine de ces initiatives sur la Côte Basque. La frontière est devenue rapidement une zone de relation: la dimension transfrontalière s'affirme par rapport aux cadres nationaux grâce aux incitations supranationales. Les revendications d'une identité basque ne sont peut-être pas étrangères à ces initiatives.

- Dans le deuxième cas, la coopération avait démarré avant 1993. Des cadres indépendants par rapport aux collectivités existantes sont créés à l'échelle de l'agglomération (p. ex. nom, structure d'encadrement). En revanche, l'intégration spatiale n'est intense que pour certaines variables. Deux coopérations ont été initiées par des "acteurs locaux» dans les années 1960 ou 1970 (Bâle et Genève), mais ont été menées à un niveau régional (Regio Basiliensis, Comité Franco-Genevois). Le Projet Européen de Développement de Longwy (PED) a été élaboré à partir de 1984 par trois Etats (Belgique. France, Luxembourg) et par la Commission Européenne dans un contexte de crise économique et sociale.

- Le troisième cas correspond à des espaces urbains où la démarche politique se veut avant tout opératoire. Une logique de projet est à l'œuvre: des lieux sont aménagés dans le cadre de la coopération à proximité des frontières notamment. Les collectivi- 

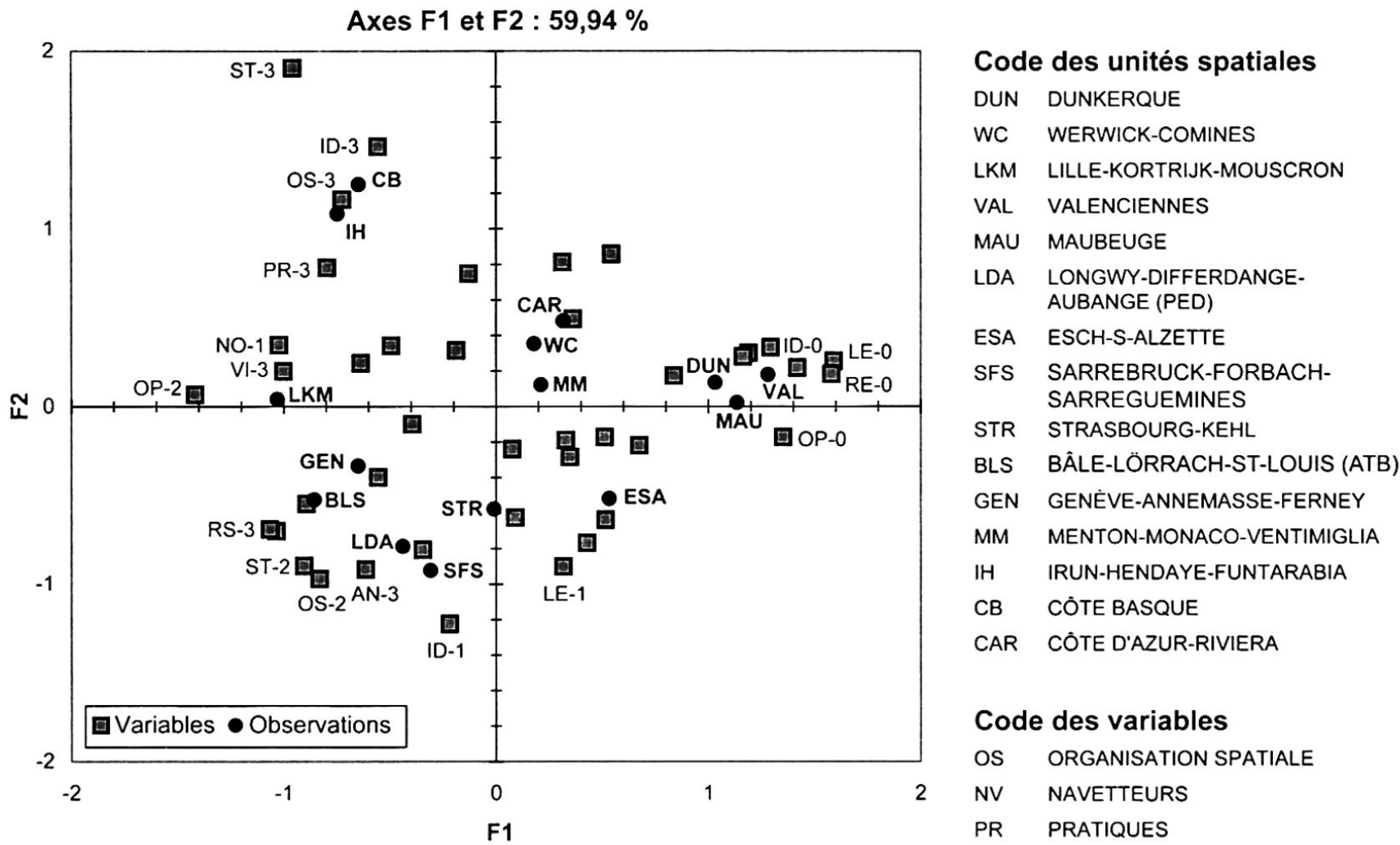

\section{Code des variables}

$\begin{array}{ll}\text { OS } & \text { ORGANISATION SPATIALE } \\ \text { NV } & \text { NAVETTEURS } \\ \text { PR } & \text { PRATIQUES } \\ \text { LE } & \text { LIEUX } \\ \text { ID } & \text { IDENTITE } \\ \text { RE } & \text { RESEAU } \\ \text { AN } & \text { ANCIENNETE } \\ \text { NO } & \text { NOM } \\ \text { VI } & \text { VISION } \\ \text { ST } & \text { STRUCTURE } \\ \text { RS } & \text { RESSOURCES } \\ \text { OP } & \text { OPERATIONNEL }\end{array}$

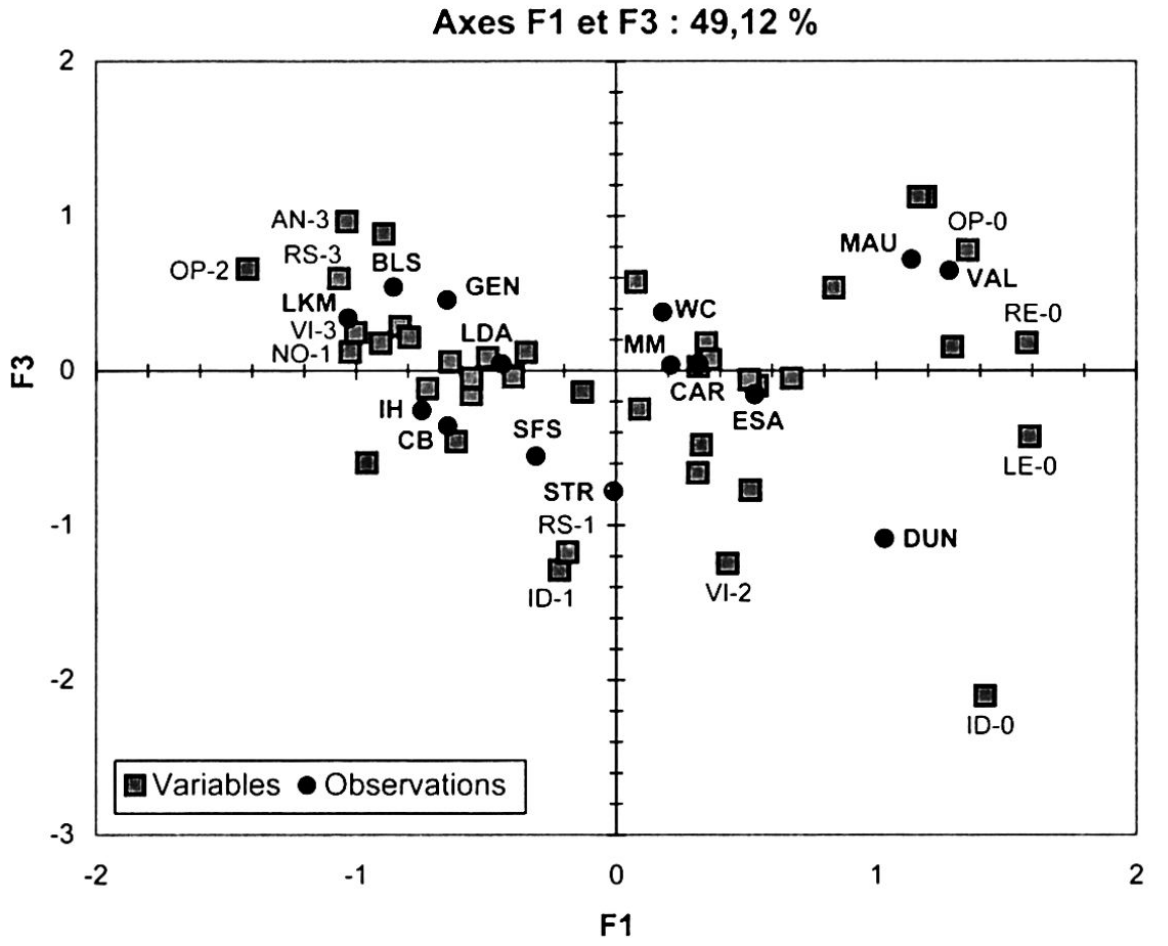

F1 : $41,97 \%$

- valeurs négatives : faible intégration spatiale

- valeurs positives : intégration territoriale

F2 : $17,97 \%$

- valeurs négatives : différentiels élevés (navetteurs)

- valeurs positives : pratiques, identités transfrontalières

F3 : $7,14 \%$

Fig. 2: Graphiques des axes factoriels de l'analyse des correspondances multiples

Faktorachsen der multiplen Korrespondenzanalyse

Factoral axis of multi-varied correspondence analysis

Graphiques: B. ReITEL 


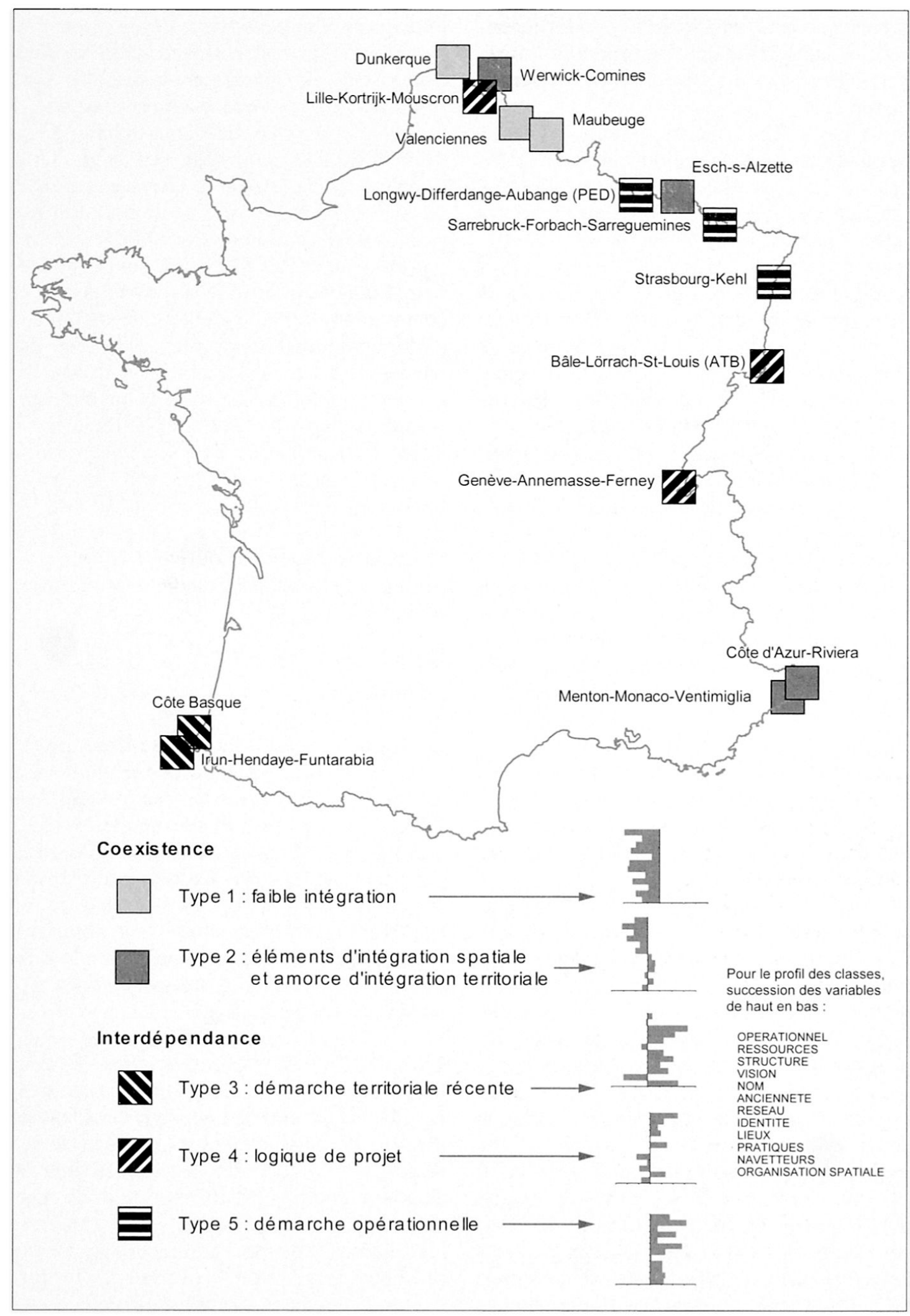

Fig. 3: Type d'intégration des agglomérations transfrontalières «françaises» Integrationstyp der «französischen» grenzüberschreitenden Agglomerationen Integration type of «French» cross-border agglomerations

Sources: Mission Opérationnelle Transfrontalière (MOT), sites internet des collectivités, Institut National de la Statistique et des Etudes Economiques (INSEE); concept: B. Reitel; graphique: Centre de Recherches Sur les Economies, les Sociétes, les Arts et les Techniques (CRESAT), J.-P. Droux 
tés sont entrées dans une logique d'interdépendance tout en conservant leur autonomie: l'encadrement transfrontalier est souple et les ressources restent dissociées.

Dans les trois types, l'intégration spatiale est forte, mais il est difficile de dissocier ce qui relève des propriétés strictes de la frontière (différenciation) et ce qui relève des effets de polarisation (centralités), les deux se confondant parfois (navetteurs).

En fin de compte, la frontière terrestre n'est plus un objet d'affirmation et de régulation de l'Etat central: cette fonction est à présent dévolue aux frontières de l'Espace Schengen. La frontière étudiée est devenue un objet de relation grâce aux politiques de l'UE, mais elle ne joue pas de la même manière dans toutes les agglomérations. Même si toutes les collectivités n'ont pas engagé un processus de coopération, les programmes Interreg ont une réelle incidence: il ne s'agit plus que d'une question de temps.

Dans la plupart des agglomérations, le processus d'ouverture de la frontière a renforcé les interactions. Les différences entre les systèmes nationaux n'ont pas disparu pour autant: ils ont permis à de nombreux habitants de profiter des opportunités. La croissance des flux transforme la frontière en interface, ce qui renforce l'intégration spatiale et incite les collectivités à accroître leurs relations (O'Dowd 2002), d'autant plus que la décentralisation a renforcé leur pouvoir dans la partie française. Les pouvoirs locaux renforcent leurs coopérations dans un contexte où les différenciations semblent plus actives que jamais.

La dimension transfrontalière dépend aussi largement des capacités d'initiatives des collectivités locales. La démarche de territorialisation qui est à l'œuvre dans certaines agglomérations, même si elle est incomplète, provient de la présence de pouvoirs publics disposant de larges compétences, des ressources financières et techniques, et pour lesquels la frontière constitue un enjeu. La proximité entre frontière et pouvoir politique est un élément prédisposant à la mise en place d'une stratégie intégrant une dimension transfrontalière et ceci s'observe dans trois cas. D'une part, en tant que micro-Etats, Monaco et le Luxembourg ont une perception particulière de la frontière. D'autre part, les Etats fédérés disposent de très larges compétences: Genève et Bâle présentent chacune les caractéristiques d'une ville-Etat qui se pense dans le contexte d'un territoire très urbanisé bordé de frontières (internes et externes). L'Eurocité s'inscrit dans un cadre similaire (autonomie basque en Espagne). Le troisième cas correspond à des villes de grande taille, inscrites dans des réseaux internationaux. Métropoles régionales ou de niveau européen (Rozenblat \& Cicille 2003), elles disposent de larges ressources (financières, techniques, humaines) pour penser leur développement futur dans laquelle la dimension transfrontalière s'insère grâce à une stratégie spatiale multi-scalaire. Cette dimension est visible à Sarrebruck (en tant que capitale de la Sarre) et à San Sebastian (souhait de "rattrapage» des villes d'Europe du Nord). L'agglomération trinationale de Bâle (ATB) et Genève entrent à nouveau dans ce cas de figure (le Canton peut être assimilé à une forme d'autorité métropolitaine). C'est le seul cas où des collectivités françaises sont également concernées: Strasbourg et Lille disposent toutes deux d'une communauté urbaine, c'est-à-dire de l'une des formes d'intercommunalité les plus élaborées dans le système français (Jouve \& LeFÈvre 2004). Dans toutes les agglomérations de dimension métropolitaine, la présence d'un pôle politique stimule les autres collectivités frontalières. Une stratégie visant à l'exploitation de l'image du pôle urbain principal est alors à l'œuvre (p. ex. Kehl envers Strasbourg, Annemasse envers Genève) (Moulle 2002; Reitel 2006). Les collectivités semblent surtout vouloir utiliser la frontière comme une ressource, en combinant différenciation et relation.

\section{Conclusion}

La frontière est un élément fondamental et paradoxal de l'organisation spatiale des agglomérations transfrontalières: elle est à la fois un frein à travers les propriétés d'affirmation et de régulation et un élément d'intégration à travers la propriété de différenciation qui induit des relations. Dans un contexte d'amoindrissement des deux premières propriétés, les interactions transfrontalières augmentent: on observe le passage d'une situation de coexistence à celle d'interdépendance, c'est-à-dire un accroissement des propriétés de différences et de relations.

Trois cas sont alors observés. Dans le premier cas, la dimension territoriale est faible: la frontière joue un rôle de filtrage entre les collectivités. Dans le deuxième cas, les interdépendances sont basées avant tout sur le respect de l'autonomie des collectivités: la frontière constitue une objet d'affirmation et de régulation qui est exercé par les pouvoirs locaux tout en intensifiant les relations (Strasbourg, Sarrebruck). Le troisième cas présente l'intégration territoriale la plus forte: la frontière est perçue et est utilisée comme une ressource, une combinaison de différenciation et de relations.

Les frontières sont devenues des objets d'articulation là où des dynamiques d'intégration territoriale sont en œuvre: cela provient de l'importance des dynamiques transfrontalières (intégration spatiale) et aux efforts consentis par l'UE et les Etats. Plus que n'importe quel autre espace, les agglomérations sont assimilées à 
des «milieux transfrontaliers» (MARTINEZ 1994) qui se définissent en fonction des interactions existant entre les territoires urbains de part et d'autre de la frontière. Elles constituent aussi des «territoires d'entre-deux» qui servent ainsi de passerelles entre des territoires nationaux.

Les grandes cités possèdent un potentiel élevé d'intégration: du fait de la concentration des ressources financières, politiques et humaines et de leur proximité de la frontière, elles sont en mesure de percevoir des enjeux, de penser la frontière et son dépassement et d'élaborer une stratégie de territorialisation transfrontalière. Le temps demeure une composante essentielle: si on considère le processus de construction européenne comme inéluctable, quel temps faudra-t-il pour consolider l'intégration territoriale à l'échelle de ces agglomérations?

\section{Bibliographie}

Anderson, M. (1983): Frontier regions in Western Europe. - Totowa: Frank Cass.

ANDERSON, M. (1996): Frontiers: territory and state formation in the modern world. - Cambridge: Cambridge University Press.

Arbaret-Schulz, C., Beyer, A., Piermay, J.-L., Reitel, B., Selimanovski, C., Sohn, C. \& P. Zander (2004): La frontière, un objet spatial en mutation. - http://www. espacestemps.net/document842.html 15.12.2005.

BERGIER, J.-F. (1983): Histoire économique de la Suisse. - Paris: Armand Colin.

Brunet, R. (1997) Territoires de France et d'Europe. Raisons de géographes. - Paris: Belin.

CARPEntier, F.G. (2005): Classification ascendante hiérarchique. - http://geai.univ-brest.fr/ carpenti/20042005/PSRS83B-4.pdf

COURLET, C. (1988): La frontière: coupure ou couture? - In: Economie et humanisme 301: 5-12.

DENIS, C. (2002): Construction et image de la frontière entre les Pays-Bas et la France. Une réflexion historique sur la relation entre frontière et ville frontalière. - In: Reitel, B., Zander, P., Piermay, J.-L. \& J.-P. RENARD (éds): Villes et frontières. - Paris : Economica: 177-187.

Donnan, H. \& T. WiLson (1999): Borders: frontiers of identity, nation and state. - Oxford: Berg.

EHLERS, N. (2002): Selling the border - twin towns on a border. The case of Kerkrade and Herzogenrath. - In: Die Erde 1:37-54.

Escoffier, B. \& J. Pages (1988): Analyses factorielles simples et multiples. Objectifs, méthodes et interprétation. - Paris: Dunod.

Foucher, M. (1986): Fronts et frontières. Un tour du monde géopolitique. - Paris: Fayard.

Foucher, M. (2000): La république européenne. - Paris: Belin.
François, J.-C., Grasland. C. \& R. Le Goix (2002): L'espace compte. - In: L'Espace géographique 4:355-356.

Ginet, P. (1994): Les conséquences du décloisonnement frontalier pour la Métropole lilloise. - Lille: Thèse, UFR de Géographie, Université Scientifique et Technique de Lille (USTL).

GRADUS, Y. \& H. Lithwick (2000): Developing frontier cities. Global perspectives, regional contexts. - Dordrecht: Kluver.

Guichonnet, P.\& C. RafFestin (1974): Géographie des frontières. - Paris: Presses Universitaires de France.

Jouve, B. (1994): Urbanisme et frontières. Le cas franco-genevois. - Paris: L'Harmattan.

Jouve. B. \& C. Lefèvre (2004): Horizons métropolitains. - Lausanne: Presses Polytechniques et Universitaires.

Kotek, J. (1996): L'Europe et ses villes-frontières. - Bruxelles: Complexe.

Leimgruber, W. (1989): The perception of boundaries. Barriers or invitation to interaction? - In: Regio Basiliensis 30, 2-3: 49-59.

LENTACKER, F. (1974): La frontière franco-belge. Etude géographique des effets d'une frontière internationale sur la vie de relations. - Lille: Morel \& Corduant.

Marcou, G. \& H. JacQuot (éds) (1997): L'urbanisme transfrontalier. Droits et pratiques. - Paris: L'Harmat$\tan$.

Martinez, O. (1994): The dynamics of border interaction. New approaches to border analysis. - In: ScHoFIELD, C. (éd.): Global boundaries. World boundaries. - Londres: Routledge: 4-16.

Menjot, D. (1996): Les villes-frontière. Moyen-Age, époque moderne. - Paris: L'Harmattan.

MEYER, J. (1984): Les deux grandes villes militaires du Nord-Est de conquête récente: Strasbourg et Lille. In: Etudes sur les villes en Europe occidentale (milieu du XVII" siècle à la veille de la Révolution française). - Paris: Sedes: 224-245.

Mission Opérationnelle Transfrontalière (MOT, 2003): Les agglomérations transfrontalières. - In: Cahiers de la MOT 3: 8-13.

Moriconi-Ebrard, F. (2002): Base Geopolis. Pour comparer les villes du monde. - http://www.geo. univ-avignon.fr/Site \%20Avignon/pages/labo/ index\%20geopolis.html 25.10.2006.

Moulle, F. (2002): L’agglomération transfrontalière genevoise. Acteurs, stratégies et fonctions internationales. - In: Reitel, B., Z ANDER, P., Piermay, J.-L. \& J.-P. Renard (éds): Villes et frontières. - Paris: Economica: 105-123.

Newman, D. (2003): On borders and power. A theoretical framework. - In: Journal of borderlands studies 18, 1: 13-25.

Nordman, D. (1998): Frontières de France. De l'espace au territoire ( $\mathrm{XVI}^{\mathrm{e}}$-XIX $\mathrm{X}^{\mathrm{c}}$ siècles). - Paris: Gallimard.

O'DowD, L. (2002): Transnational integration and 
cross-border regions in the European Union. - In: Anderson, J. (éd.): Transnational democracy. Political spaces and border crossings. - Londres: Routledge: 111-128.

PAASI, A. (1996): Territories, boundaries and consciousness. The changing geographers at the finnishrussian border. - Chichester: John Wiley.

Piermay, J.-L. (2005): La frontière et ses ressources: regards croisés. - In: AnTHEaume, B. \& F. GiRaut (éds): Le territoire est mort. Vive les territoires! - Paris: Institut de recherche pour le développement (IRD): 203222.

Pollmann, C. (1999): La frontière: horizon indépassable de l'humanité ou pouvoir objectivé? - In: Revue de droit public 2: 481-499.

Prescotr, J.R.V. (1978): Boundaries and frontiers. - Londres: Methuen.

Raffestin, C. (1980): Pour une géographie du pouvoir. - Paris: Librairies techniques.

RafFestin, C. (1986): Eléments pour une théorie de la frontière. - In: Diogène 134: 3-21.

RatTI, R. \& S. Reichman (1993): Theory and practice of transborder cooperation. - Bâle: Helbing \& Lichtenhahn.

Reitel, B., Zander, P., Piermay, J.-L. \& J.-P. Renard (éds) (2002): Villes et frontières. - Paris: Economica.

Reitel, B. (2006): Les logiques de projet dans l'agglomération transfrontalière de Strasbourg-Kehl et l'agglomération trinationale de Bâle: vers l'émergence de nouvelles territorialités? - In: Fourny, M.-C. \& A.-L. Amilhat-Szary (dirs): Après les frontières, avec la frontière. Nouvelles dynamiques transfrontalières en Europe. - Paris: Editions de l'Aube: 151-167.

Renard, J.-P. \& P. Picouet (1993): Frontières et territoires. - Paris: La Documentation française.

Rozenblat, C. \& P. Cicille (2003): Les villes européennes. Analyse comparative. - Paris: La Documentation française.

SAez, G., Leresche, J.-P. \& M. Bassand (1997): Gouvernance métropolitaine et transfrontalière. Action publique territoriale. - Paris: L'Harmattan.

SANDTNER, M. \& S. Eder (2000): Staatsgrenzen in der Regio Trirhena. Barriere oder Stimulus? - In: Regio Basiliensis 41, 1: 15-26.

SCHNeIDER-Sliwa, R. (2002): Vers une région sans frontières? - In: Revue Géographique de l'Est 42, 1-2: 5-8. Staehlin, A. (1979): Bâle ville frontière: quelques réflexions. - In: LA BACONNIÈRE (éd.): Frontières et contacts de civilisation. Actes de colloque franco-suisse, Besançon-Neuchâtel 1977. - Neuchâtel: La Baconnière: $149-158$.

WAACK, C. (2000): Stadträume und Staatsgrenzen. Geteilte Grenzstädte des mittleren und östlichen Europas im Kontext lokaler Alltagswelten, nationaler Politik und supranationaler Anforderungen. - Leipzig: Institut für Länderkunde.

Wastl-Walter, D. \& C. Kofler (2000): European integration and border-related institutions: a practical guide. - In: Journal of borderlands studies 15, 1: 85106.

\section{Résumé: Les agglomérations transfrontalières: des systèmes urbains en voie d'intégration? Les espaces urbains de la «frontière» du territoire français}

En tant que limite politique signifiante, la frontière a joué un rôle majeur dans la construction du territoire national de la France. Elle n'a cependant pas été qu'une barrière: une quinzaine d'agglomérations transfrontalières s'y sont formées. L'objectif de cet article consiste à évaluer le degré d'intégration de 15 agglomérations situées sur les frontières de France, dans une perspective de construction européenne et de dévaluation des frontières nationales. L'étude s'appuie sur deux dimensions: l'une spatiale (organisation, pratiques), l'autre territoriale (p. ex. politiques de coopération, projets).

Les résultats d'une analyse multivariée montrent que l'importance de la dimension territoriale est liée aux formes de l'intégration spatiale. En s'appuyant sur les propriétés des frontières décrites par RAFFESTIN (1986) et sur le modèle des interactions sociales dans les espaces transfrontaliers proposé par MARTINEZ (1994), il apparaît que la construction européenne se traduit au niveau local par un passage d'une situation de coexistence (des territoires urbains juxtaposés, situés dans des cadres nationaux contigus) à une situation d'interdépendance (des territoires urbains appartenant à une entité supranationale, entre lesquels s'effectuent des interactions croissantes). Les frontières sont devenues des objets d'articulation: les agglomérations peuvent être assimilées à des territoires de «l'entre-deux». Dans ce contexte, les grandes cités possèdent un potentiel élevé d'intégration: la concentration des ressources financières, politiques et humaines et la proximité de la frontière leur permet de percevoir des enjeux, de penser la frontière et son dépassement et d'élaborer une stratégie de territorialisation transfrontalière.

\section{Zusammenfassung: Grenzüberschreitende Agglomera- tionen: Städtische Systeme auf dem Weg zur Integration? Die Stadträume entlang der französischen Grenze}

Die Grenze als politisch signifikante Linie spielte eine große Rolle in der Konstruktion des französischen nationalen Territoriums. Diese Grenze war aber nicht nur eine Schranke: grenzüberschreitende Agglomerationen haben sich entwickelt. Diese städtischen Räume sind in ihrer Funktion und ihrer Verwaltung von der Grenze geprägt. Das Ziel dieses Artikels ist, im Rahmen des europäischen Integrationsprozesses den Integrationsgrad von fünfzehn grenzüberschreitenden Agglomerationen Frankreichs zu ermitteln: die räumliche Dimension sowie die politische (territo- 
riale) Dimension werden in Betracht gezogen. Es wird die Rolle der Grenzen in diesen Räumen bestimmt.

Die Ergebnisse der multivariaten Analyse zeigen, dass die territoriale Dimension am höchsten ist, wenn der räumliche Integrationsgrad stark ist. Das Modell der sozialen Interaktionen der Grenzräume (MARTINEZ 1994) und die Eigenschaften von Grenzen (RAFFESTIN 1986) werden als Untersuchungsraster benutzt. Im europäischen Integrationsprozess haben sich die Beziehungen der Stadträume auf lokaler Ebene von einem Koexistenz-Zustand hin zu gegenseitiger Abhängigkeit entwickelt: nebeneinander liegende städtische Gebiete, die sich aber in Nachbarstaaten befinden, wuchsen zu städtischen Gebieten in einem übernationalen Raum zusammen. Die Grenzen sind Artikulationsgegenstände geworden: Die Stadträume können als «in-between territories» betrachtet werden. Die großen, grenzüberschreitenden Agglomerationen besitzen höhere Integrationspotentiale: In ihnen konzentrieren sich finanzielle und politische Mittel sowie Humankapital und, dank ihrer Nähe zur Grenze, können Herausforderungen angepackt und komplexe territoriale Strategien entwickelt werden.

\section{Abstract: Cross-border agglomerations: urban sys- tems on the path to integration? Urban spaces on the French border}

The border, as a significant political limit, has a powerful role to play in the construction of what might be called the French national territory. This demarcation line is, however, not as solid as it seems: cross-border urban spaces have been able to develop and their functionality, spatial organization and management have, to a large extent, been linfluenced by it. The integration processes currently to be seen in Europe have led to greater attention being paid to dismantling of borders and increased initiation of cross-border cooperation programmes (such as Interreg). This article therefore aims to assess the changing roles of borders by determining the degree of integration of fifteen cross-border urban spaces located in the proximity of the boundary of the French national territory. Attention is paid in particular to interaction at two levels, at the spatial level (organization of the urban area, spatial practices) and at the territorial level (projects, political cooperation).
The first results of the multi-varied analysis indicate that territorial integration depends to a great part on the intensity of spatial integration. The pattern of social interaction in cross-border areas of MARTINEZ (1994) and the border characteristics of RAFFESTIN (1986) formed the basis of the analysis grid. The process of European unification has led to situations where coexistence of border urban spaces have developed into an interdependence of urban neighbours in European space. Borders have been given a voice; crossborder urban spaces have become «in-between territories». The article concludes that large cities have the greatest potential for cross-border integration. Due to the concentration of financial, political and human resources in such centres, as well as their proximity to the border, these cities have the necessary power to meet the challenges that face them and to initiate processes supporting the development of complex territorialisation strategies.

Dr. Bernard Reitel, Centre de recherche sur les économies, les sociétés, les arts et les techniques (CRESAT), Université de Haute-Alsace, 10, rue des Frères Lumière, F-68093 Mulhouse Cedex, France.

e-mail: bernard.reitel@uha.fr

\section{Manuskripteingang/received/manuscrit entré le} 3.7.2006

Annahme zum Druck/accepted for publication/accepté pour l'impression: 25.2.2007 